\title{
Sensitivity to a new right-handed charged current in polarized hadronic collisions at RHIC
}

\author{
P. Taxil and J.M. Virey ${ }^{1}$ \\ Centre de Physique Théorique*, C.N.R.S. - Luminy, Case 907 \\ F-13288 Marseille Cedex 9, France \\ and \\ Université de Provence, Marseille, France
}

\begin{abstract}
In the context of general $S U(2)_{L} \otimes S U(2)_{R} \otimes U(1)$ models a relatively light $W^{\prime}$ is still allowed in some scenarios with a heavy right-handed neutrino and a quark mixing matrix $U^{R}$ close to unity. We explore the consequences of the presence of this new charged current on the parity violating spin asymmetries which could be induced in one-jet inclusive production in polarized proton-proton or proton-neutron collisions. Such measurements could be performed within a few years at the Brookhaven Relativistic Heavy Ion Collider (RHIC) running part of the time as a polarized hadronic collider.
\end{abstract}

PACS Numbers : 12.60.Cn; 13.87.-a; 13.88.+e; 14.70.Pw

Key-Words : New Gauge bosons, Jets, Polarization.

Number of figures : 2

April 1997

CPT-97/P.3469

anonymous ftp or gopher : cpt.univ-mrs.fr

*Unité Propre de Recherche 7061

${ }^{1}$ Moniteur CIES and allocataire MESR

E-mail : Taxil@cpt.univ-mrs.fr ; Virey@cpt.univ-mrs.fr 


\section{Introduction}

The possibility of new charged current interactions for the right-handed fermions has been considered for a long time as one of the direct extensions of the electroweak Standard Model (SM). The simplest example is the left-right symmetric model based on the gauge group $S U(2)_{L} \otimes S U(2)_{R} \otimes U(1)_{B-L}$ [1] which implies the presence of three new gauge bosons, two charged $W^{\prime \pm}$ and one neutral $Z^{\prime}$.

It is well known that the new charged bosonic sector is more severely bounded by low-energy data than the neutral one. The most severe constraints are coming from the $K_{S}-K_{L}$ mass difference $\Delta m_{K}$. These constraints, however, rely on some specific assumptions (for a nice review see [2] and references therein).

For the symmetric models with $g_{L}=g_{R}$, and if one assumes an equal magnitude for the left-handed and right-handed quark mixing matrix elements $U_{i j}^{L}$ and $U_{i j}^{R}$ ("manifest L-R symmetry"), the bound which can be obtained on the mass of the heavy right-handed charged boson is rather stringent : $M_{W^{\prime}} \geq 1.4-2.5 \mathrm{TeV}$, with the exact value depending on the values of the QCD enhancement factor [3]. The limits are the same for "pseudomanifest L-R symmetry" that is if $\left|U^{L}\right|=\left|U^{R}\right|$. Note that the approximations in the estimates of the hadronic matrix elements introduce some uncertainty : an approach based on the QCD sum rules lowers this bound down to $700 \mathrm{GeV}$ [ [I] . In the same time the $W-W^{\prime}$ mixing angle is also severely bounded from universality : $|\xi|<0.003$. However, if more freedom is allowed on the gauge couplings ("general left-right models" with $g_{L} \neq g_{R}$ ) and especially on the values of the matrix elements $U_{i j}^{R}$, most of the constraints can be evaded. The weakest (90\% C.L.) bound for $M_{W^{\prime}}$ advocated in [2] correspond to $U^{R} \approx I$ (identity), it corresponds to :

$$
M_{W^{\prime}} / \kappa \geq 300 \mathrm{GeV}
$$

where $\kappa=g_{R} / g_{L}$. Concerning the mixing angle $\xi$ one still gets a bound in the $1 \%$ range: $\kappa|\xi|<0.013$. Following grand unification arguments, it is usually assumed that $0.55 \leq \kappa \leq 1$ which can give us a $W^{\prime}$ as light as $\approx 170 \mathrm{GeV}$. Note that, with the present value of the top mass, these relatively old limits can be lowered by at least 10\% [2, 5].

Concerning direct searches, in $\bar{p} p$ collisions, with the hypothesis that the $\nu_{R}$ is sufficiently light to allow the decay $W^{\prime} \rightarrow l_{R} \nu_{R}$, CDF obtained the limit : $M_{W^{\prime}}>652 \mathrm{GeV}$ (at $95 \%$ C.L.) [6]. This limit is obtained in the leptonic channel in case of $\kappa=1$ and $U^{R}=U^{L}$. From low energy muon decay analysis, with the same assumptions, the bound is $406 \mathrm{GeV} \mathrm{[7].} \mathrm{On} \mathrm{the} \mathrm{other} \mathrm{hand,} \mathrm{if} \mathrm{the} W^{\prime}$ leptonic decay is forbidden, due to a very large mass value for the $\nu_{R}$, the constraints are much weaker since the direct search in hadronic collisions is then restricted to the two-jet decay channel (the $W Z$ decay channel will also be promising but only at LHC [8]). In this situation, the mass domain excluded by UA2 9] $100<M_{W^{\prime}}<250 \mathrm{GeV}$ (at 90\% C.L.) has been very recently extended by CDF [10]: $300<M_{W^{\prime}}<420 \mathrm{GeV}$ (at 95\% C.L.). Both experiments assumed $\kappa=1$. From CDF published data, one can infer that the lower bound on $W^{\prime}$ is disappearing if the value of $\kappa$ is reduced below $\kappa=0.95$. 
Actually, due to the peculiarities of the two-jet channel, a window is still open in the region below $100 \mathrm{GeV}$. This region is not strictly forbidden neither by $\Delta m_{K}$ nor by bounds from double-beta decay as soon as $i$ ) the right-handed neutrino is a massive Dirac neutrino $i$ ) extreme fine tuning of the $U^{R}$ parameters (three angles and six phases) is allowed to get a cancellation between terms involved in the calculation of $\Delta m_{K}$ [2]. The existence of this window has been noticed at the recent HERA workshop [11] : at HERA, in this case, high luminosities should allow a significant discovery potential.

Many studies have been devoted to the sensitivity of the ongoing Tevatron searches for the high $W^{\prime}$ mass domain, assuming that the missing $E_{T}$ leptonic decay mode is accessible and dominant [5, 12]. On the other hand, direct detection of the $W^{\prime}$ in the two-jet channel will probably remain difficult and full of the uncertainties inherent to this channel.

From now, we will place ourselves in the scenario where a very massive right-handed neutrino forbids the leptonic decay. As discussed above, in the case where $U^{R} \approx I$, a relatively light $W^{\prime}$ is still allowed, with various possible windows for the values of its masses, depending on the value of $\kappa$.

In fact, the presence of such $W^{\prime}$ exchanges in the quark-(anti)quark scattering subprocesses could induce some other kinds of deviations from the SM expectations, at subenergies $\sqrt{\hat{s}}$ below $M_{W^{\prime}}$. Due to the right-handed structure of the new current, it should give rise to particular parity violating (PV) spin effects and this should be exploited. Since, in the context of hadronic collisions it is quite hopeless to analyze the net helicity of an outgoing particle or jet, polarized beams are needed to build an initial-state spin asymmetry. Indeed, we have shown recently that, in polarized $p p$ collisions, the presence of a hadrophilic $Z^{\prime}$ [13] and/or a new PV contact interaction between quarks [14] could yield some deviations from the spin asymmetry in one-jet inclusive production which is due to SM electroweak (EW) bosons exchanges.

Theses analyses were performed in the context the Relativistic Heavy Ion Collider (RHIC) which will be run part of the time as a polarized hadronic collider. This program 15] will start in a few years from now, with high intensity polarized proton beams and a center of mass energy from $200 \mathrm{GeV}$ (for the first run) up to 500-600 GeV. The possibility of accelerating polarized ${ }^{3} \mathrm{He}$ nuclei, which has been discussed recently [16, 17], will open some new perspectives since polarized $p n$ collisions will be allowed.

In the following we will discuss the influence of the new $W^{\prime}$ on the one-jet PV spin asymmetry $A_{L L}^{P V}$ in polarized $p p$ and $p n$ collisions at the planned RHIC energies and luminosities. We first present the ingredients entering into the calculation of the asymmetry $A_{L L}^{P V}$, then we give the limits which could be obtained on the parameter space $\left(\kappa, M_{W^{\prime}}\right)$ of the new right-handed charged sector, given the sensitivity of the polarized RHIC experiments. 


\section{Double helicity PV asymmetry in one-jet inclusive production}

For the inclusive process $H_{a} H_{b} \rightarrow$ jet $+X$, when both beams can be polarized (this is the case at RHIC), one defines a double helicity PV asymmetry :

$$
A_{L L}^{P V}=\frac{d \sigma_{a(-) b(-)}-d \sigma_{a(+) b(+)}}{d \sigma_{a(-) b(-)}+d \sigma_{a(+) b(+)}}
$$

where the signs \pm refer to the helicities of the colliding hadrons. From now, $d \sigma_{a\left(h_{a}\right) b\left(h_{b}\right)}$ will mean the cross section in a given helicity configuration $\left(h_{a}, h_{b}\right)$, for the production of a single jet at a given transverse energy $E_{T}$ and pseudorapidity $\eta$ :

$$
d \sigma_{a\left(h_{a}\right) b\left(h_{b}\right)} \equiv \frac{d^{2} \sigma^{\left(h_{a}\right)\left(h_{b}\right)}}{d E_{T} d \eta}
$$

In the following, we integrate $d \sigma$ over a pseudorapidity interval $\Delta \eta=2.6$ or 1.0 (see below) centered at $\eta=0$, and over an $E_{T}$ bin which corresponds to a jet energy resolution of $10 \%$.

Any helicity dependent hadronic cross section is obtained by convoluting appropriately the subprocess cross sections $d \hat{\sigma}_{i j}^{\lambda_{1}, \lambda_{2}} / d \hat{t}$, which depend upon the parton helicities $\lambda_{1}$ and $\lambda_{2}$, with the polarized quark and/or antiquark distributions evaluated at some scale $\mu^{2}$ : $q_{i \pm}\left(x, \mu^{2}\right)$ and $\bar{q}_{i \pm}\left(x, \mu^{2}\right)$ (explicit formulas can be found in 18, 19]). Here, $q_{i \pm}$ means the distribution of the polarized quark of flavor $i$ having its helicity parallel $(+)$ or antiparallel (-) to the parent hadron helicity. It is usual to define $\Delta q_{i}=q_{i+}-q_{i-}$. The chosen $\mu^{2}$ value is $\mu^{2}=E_{T}^{2}$, we have checked that changing this choice has no visible influence on our results.

We follow the notations of [19] where :

$$
\frac{d \hat{\sigma}_{i j}^{\lambda_{1}, \lambda_{2}}}{d \hat{t}}=\frac{\pi}{\hat{s}^{2}} \sum_{\alpha, \beta} T_{\alpha, \beta}^{\lambda_{1}, \lambda_{2}}(i, j)
$$

$T_{\alpha, \beta}^{\lambda_{1}, \lambda_{2}}(i, j)$ denoting the matrix element squared with $\alpha$ boson and $\beta$ boson exchanges, in a given helicity configuration for the involved partons $i$ and $j$.

QCD dominates the unpolarized cross section and it is not difficult to incorporate into the calculation the tiny EW terms, their interference with QCD amplitudes [20] and also the Non Standard $W^{\prime}$ terms with a $W^{\prime}$ coupling to quarks of the form

$$
\imath \frac{g_{R}}{\sqrt{2}} \bar{q}_{i} \frac{1}{2}\left(1+\gamma_{5}\right) \gamma_{\mu} W^{\prime \mu} U_{i j}^{R} q_{j}
$$

These latter have a small influence on the unpolarized cross section in the range of parameters we consider. At RHIC energies it is then hopeless to isolate a bump in the dijet mass spectrum if $M_{W^{\prime}}$ lies in the range which is still allowed. 
Concerning $A_{L L}^{P V}$, the leading order (LO) SM contribution is known for a long time 21]. It comes essentially from interferences between QCD and standard EW PV amplitudes (see [19] for the correct expressions for the relevant $T_{\alpha, \beta}$ 's). It is raising with $E_{T}$ at large $E_{T}$ due to the increasing influence of $q q$ scattering [22] and it was reestimated recently using modern $\Delta q_{i}$ 's in [14, 13] for $\vec{p} \vec{p}$ scattering.

Note that it can be advocated that a $\mathrm{LO}$ calculation of $A_{L L}^{P V}$ gives a quite good estimate since, QCD being helicity conserving in the limit of massless quarks, one does not expect a significant influence of NLO corrections on the spin asymmetries. Indeed, when NLO calculations are available as is the case for inclusive prompt photon production, it has been stressed that the spin asymmetries were less influenced by the NLO corrections than the individual polarized or unpolarized cross sections [23].

Let us now concentrate on the effect of the new right-handed current.

In the $E_{T}$ range of interest the contribution of quark-quark scattering $q_{i} q_{j}(i \neq j)$ dominates over terms involving antiquarks (these latter are carefully taken into account in the full calculation). In short notations $A_{L L}^{P V}$ is then given by the expression :

$$
A_{L L}^{P V} \cdot d \sigma \simeq-\sum_{i, j} \int T_{g, W^{\prime}}^{++}(i, j)\left[q_{i}\left(x_{1}, \mu^{2}\right) \Delta q_{j}\left(x_{2}, \mu^{2}\right)+\Delta q_{i}\left(x_{1}, \mu^{2}\right) q_{j}\left(x_{2}, \mu^{2}\right)+(i \leftrightarrow j)\right]
$$

where $T_{g, W^{\prime}}^{++}(i, j)$ is the term originating from the interference between one gluon exchange and the $W^{\prime}$ exchange amplitudes for right-handed quarks (taking care of color rules and crossing symmetry) :

$$
T_{g, W^{\prime}}^{++}(i, j)=\frac{4}{9} \alpha_{s}\left(\mu^{2}\right) \frac{g_{R}^{2}}{\pi}\left|U_{i j}^{R}\right|^{2} \frac{\hat{s}^{2}}{\hat{u}_{W^{\prime}} \hat{t}}
$$

where $\hat{u}_{W^{\prime}}=\hat{u}-M_{W^{\prime}}^{2}$.

Since the scattering of valence quarks of different flavours is the dominant process, it is easy to understand why proton-neutron collisions will be preferred to the more familiar proton-proton case.

For $\vec{p} \vec{p}$ collisions, when charged bosons $W$ (or $W^{\prime}$ ) are involved and if one neglects the heavy flavour content of the nucleon, only the expressions $u \Delta d+d \Delta u$ will enter in eq.(6). Some very general tendencies of the polarized quark distributions are : i) $\Delta u>0, \Delta d<0$; ii) $u \Delta u \gg d|\Delta d|$; iii) $\Delta u / u>|\Delta d| / d$. Therefore, in case of $W$ $\left(W^{\prime}\right)$ exchanges, there is a partial cancellation in the $u \Delta d+d \Delta u$ term. This cancellation is much less important in case of $Z^{\circ}$ (or neutral $Z^{\prime}$ ) exchanges where the combination $u \Delta u+d \Delta d$ dominates in the corresponding formula (see [13]) thanks to the dominance ii). Note that this partial cancellation is somewhat compensated by the maximal PV in eq.(6) compared to the relatively smaller amount of PV which is present in the neutral sector (at least when the standard $Z^{\circ}$ is involved).

In $\vec{p} \vec{n}$ collisions the situation is reversed if one invokes Isospin symmetry which implies $u^{n}=d^{p}(\equiv d), d^{n}=u^{p}(\equiv u)$ and $\Delta u^{n}=\Delta d^{p}, \Delta d^{n}=\Delta u^{p}$ in the same way. The net results is that $\vec{p} \vec{p}$ collisions are well suited to see the effect of gluon-neutral gauge boson 
( $Z^{\circ}$ and/or $Z^{\prime}$ ) interferences, whereas in $\vec{p} \vec{n}$ collisions, the interferences between gluons and charged gauge bosons ( $W$ and/or $W^{\prime}$ ) are enhanced.

In the following, we will only present the results of our analysis on the most interesting channel of polarized $\vec{p} \vec{n}$ collisions.

\section{Discussion and results}

In Fig. 1 we show the asymmetry $A_{L L}^{P V}$ in polarized $\vec{p} \vec{n}$ collisions versus the transverse jet energy $E_{T}$ at a c.m. energy $\sqrt{s} \approx 500 \mathrm{GeV}$ that is for a $300 \mathrm{GeV}$ proton beam colliding with a $600 \mathrm{GeV}$ beam of polarized ${ }^{3} \mathrm{He}$ nuclei. We have chosen for illustration $M_{W^{\prime}}=100$ $\mathrm{GeV}, 300 \mathrm{GeV}$ and $400 \mathrm{GeV}$ (for $\kappa=1$ ) and $200 \mathrm{GeV}$ (for $\kappa=0.8$ ). The standard $A_{L L}^{P V}$, which is bigger than the corresponding one in $\vec{p} \vec{p}$ collisions, is shown for comparison. The error bars correspond to the statistical error for a degree of polarization $\mathcal{P}=70 \%$ (see [13, 14]) and an integrated luminosity of $800 \mathrm{pb}^{-1}$ which can be achieved in a few months running, the systematic error being negligible [17]. We have chosen an $E_{T}$ region where these errors are small : the range between $60 \mathrm{GeV}$ and $100 \mathrm{Gev}$ will dominate the analysis which follows.

This calculation has been performed for $U^{R}=I$. In fact we are not sensitive to the precise form of $U^{R}$ as long as the off-diagonal matrix elements are small. Since the $W-W^{\prime}$ mixing angle is already severely restricted (less than $\approx 1 \%$ ) we can neglect safely its effects. These results are also independent of the precise value of $m_{\nu_{R}}$ as soon as one remembers that we placed ourselves in the situation where the decay $W^{\prime} \rightarrow \nu_{R} l_{R}$ is forbidden by kinematic. We have used for consistency LO spin dependent distributions, namely the ones of GRV [24] which fit well the polarized deep-inelastic scattering data. It has to be remembered that our predictions are not affected by the present uncertainties on the polarized gluon distributions. The first part of the polarized RHIC program itself [15, 17] will greatly improve our knowledge of $\Delta q_{i}$ 's and $\Delta \bar{q}_{i}$ 's.

One can see from Fig.1 that the measurement of $A_{L L}^{P V}$ at RHIC should allow to pin down easily the presence of a right-handed $W^{\prime}$ with a mass around or below $100 \mathrm{GeV}$. In this case $A_{L L}^{P V}$ is compatible with zero because an important cancellation occurs between the standard $W$-gluon and the $W^{\prime}$-gluon interference terms. A zero $A_{L L}^{P V}$ is clearly forbidden in the SM, for any reasonable choice for the spin dependent quark distributions. With the high precision achievable at RHIC, thanks to the high luminosity, such an effect cannot be missed.

We present in Fig.2 the limits we obtain at RHIC on the parameter space $\left(\kappa, M_{W^{\prime}}\right)$. The shaded areas correspond to the two zones excluded respectively by UA2 and CDF in the two-jet channel. From the published results we have smoothly extrapolated their limits down to values of $\kappa$ below 1 . The dashed line corresponds to the "theoretical" $90 \%$ C.L. upper bound $M_{W^{\prime}}=300 \kappa$ (in GeV) according to eq. (1). Remember that this bound can be avoided if extreme fine tuning of the $U^{R}$ matrix elements is allowed as discussed in [2]. The two other lines correspond to our 95\% C.L. upper limits which can be obtained at RHIC (with the same figures for the luminosity and the energy as above) after having 
integrated over a pseudorapidity domain $\Delta \eta=1$ or $\Delta \eta=2.6$. The first case corresponds to a "minimal" detector configuration, the second one to the case where it will be possible to extend the rapidity interval by adding "end-caps" to the STAR detector at RHIC [17.

These bounds display an approximate scaling : $M_{W^{\prime}}=400 \kappa-10$ (in $\mathrm{GeV}$ ) for $\Delta \eta=1$ and $M_{W^{\prime}}=500 \kappa-30$ (in $\mathrm{GeV}$ ) for $\Delta \eta=2.6$. It is clear that the second option is preferred since it allows to probe the presence of a new $W^{\prime}$ up to $M_{W^{\prime}}=470 \mathrm{GeV}$ (for $\kappa=1$ ) instead of $390 \mathrm{GeV}$, a value already excluded by the CDF bound.

One can see that, if $\kappa=1$, there is little room for discovery except in the high mass region between $420 \mathrm{GeV}$ and $470 \mathrm{GeV}$, in the narrow window between $250 \mathrm{GeV}$ and 300 $\mathrm{GeV}$ and in the region below $100 \mathrm{GeV}$ as discussed above (ignoring the bound eq. (1) in

these last two cases). On the other hand, the precise measurement of $A_{L L}^{P V}$ should allow to cover the "small $\kappa$ " region $(0.55<\kappa<1)$ for relatively light $W^{\prime}$. For large $\kappa$ values, it is possible to probe $M_{W^{\prime}}$ up to $670 \mathrm{GeV}$, with a detector with maximal coverage. In the case of $\vec{p} \vec{p}$ collisions, the bounds from $A_{L L}^{P V}$ fall into the region already excluded by UA2. The region $M_{W^{\prime}} \leq 100 \mathrm{GeV}$ is of course still interesting in this configuration.

\section{Conclusion}

A consequence of the scenario with a very massive right-handed neutrino is the difficulty to pin down the presence of a new right-handed charged gauge boson $W^{\prime}$ which is supposed to decay only into a pair of jets with no missing energy. Looking in this channel CDF and UA2 had left open some consequent windows in the parameter space. On the other hand it has been stressed that low energy bounds on $M_{W^{\prime}}$ can be evaded, leaving open the possible existence of a light or relatively light $W^{\prime}$.

Polarized hadronic collisions offer a unique opportunity to get a handle on the interference effects which could be induced by the new charged current. Indeed, the righthanded nature of the $W^{\prime}$ introduces some disturbance on the spin asymmetry $A_{L L}^{P V}$ in inclusive one-jet production. These effects could be seen at RHIC for quite a large portion of the parameter space $\left(\kappa, M_{W^{\prime}}\right)$. Of course, a great precision is needed : at RHIC this can be achieved in a few months running thanks to the very high luminosity $\left(\mathcal{L}=2.10^{32} \mathrm{~cm}^{-2} \mathrm{~s}^{-1}\right)$ and to the high degree of beam polarization. To complete this program, the availability of polarized neutrons is necessary and this can be obtained with beams of ${ }^{3} \mathrm{He}$ or Deuteron nuclei.

Finally, if a remarkable deviation from the standard $A_{L L}^{P V}$ is observed, then it will be mandatory to perform some careful analysis to get some informations on the true nature of the new interaction. Disentangling the effect of a hadrophilic $Z^{\prime}$ from the one of a new $W^{\prime}$ or from a flavour conserving and parity violating Contact Term will not be an easy task. For this purpose, having at disposition both polarized proton and neutron beams could greatly help. These questions will be treated in more detail in a forthcoming analysis. 


\section{Acknowledgments}

We thank J. Soffer for fruitful discussions, A. Zylberstejn for information on the FNAL ex-

periments and the members of the RHIC Spin Collaboration (RSC) for useful discussions at the occasion of the recent Marseille's RSC meeting.

\section{References}

[1] J.C. Pati and A. Salam, Phys. Rev. Lett. 31, 661 (1973); Phys. Rev. D10, 275 (1974); R.N. Mohapatra and J.C. Pati, Phys. Rev. D11, 566 (1975).

[2] P. Langacker and S.Uma Sankar, Phys. Rev. D40, 1569 (1989).

[3] G. Beall, M. Bander and A. Soni, Phys. Rev. Lett. 48, 848 (1982) ; G. Ecker and W. Grimus, Nucl. Phys. B258, 328 (1985).

[4] P. Colangelo and G. Nardulli, Phys. Lett. B253, 154 (1991).

[5] T.G. Rizzo, Phys. Rev. D50, 325 (1994).

[6] F. Abe et al., Phys. Rev. Lett. 74, 2900 (1995).

[7] Particle Data Group, Phys. Rev. D54, 1, 1996.

[8] see e.g. M. Cvetic, P. Langacker and B. Kayser, Phys. Rev. Lett. 68, 2871 (1992) or P. Chiappetta, M. Deliyannis, A. Fiandrino and P. Taxil, Phys. Lett. B308, 304 (1993) and refs. therein.

[9] J. Alitti et al., (UA2 Collaboration), Nucl. Phys. B400, 3 (1993).

[10] F. Abe et al., FNAL-PUB-97/023-E hep-ex/9702004.

[11] Workshop on Future Physics at HERA 1995-1996 ; see e.g. the summary of the Beyond the Standard Model Group by H. Dreiner, H.U. Martyn, S. Ritz and D. Wyler hep-ph/9610232.

[12] T.G. Rizzo, SLAC-PUB-7365 [hep-ph/9612440, report of the New gauge Boson Subgroup of the 1996 Snowmass Summer Study on New Directions for High Energy Physics.

[13] P. Taxil and J.M. Virey, Phys. Lett. B383, 355 (1996).

[14] P. Taxil and J.M. Virey, Phys. Lett. B364, 181 (1995) ; Phys. Rev. D55, 4480 (1997).

[15] G. Bunce et al. (RHIC Spin Collaboration), Polarized protons at RHIC, Particle World, 3, 1 (1992). 
[16] Proceedings of the 12th International Symposium on High Energy Spin Physics, Amsterdam, september 1996 (to appear).

[17] RHIC Spin Collaboration meeting, Marseille, september 1996, preprint CPT96P./3400.

[18] C. Bourrely, J. Soffer, F.M. Renard and P. Taxil, Phys. Reports,177, 319 (1989).

[19] C. Bourrely, J. Ph. Guillet and J. Soffer, Nucl. Phys. B361, 72 (1991).

[20] M. Abud, R. Gatto and C.A. Savoy, Ann. Phys. (NY) 122, 219 (1979) ; U. Baur, E.W.N. Glover and A.D. Martin, Phys. Lett. B232, 519 (1989).

[21] F.E. Paige, T.L. Trueman and T.N. Tudron, Phys. Rev. D19, 935 (1979) ; J. Ranft and G. Ranft, Nucl. Phys. B165, 395 (1980).

[22] M. Tannenbaum, in Polarized Collider Workshop, J. Collins, S.F. Heppelmann and R.W. Robinett eds, AIP Conf. Proceedings 223, AIP, New York, 1990, p. 201.

[23] L.E. Gordon and W. Vogelsang, Phys. Lett. B387, 629 (1996) ; L.E. Gordon, ANLHEP-PR-96-100 hep-ph/9612270.

[24] M. Gluck, E. Reya and W. Vogelsang, Phys. Lett. B359, 201 (1995). 


\section{Figure captions}

Fig. $1 A_{L L}^{P V}$ for one-jet inclusive production, versus $E_{T}$, for polarized $p n$ collisions at RHIC at a c.m. energy of $500 \mathrm{GeV}$. Standard model expectation (plain curve), $W^{\prime}$ effects with a $W^{\prime}$ mass of $400,300,100 \mathrm{GeV}(\kappa=1)$ and $200 \mathrm{GeV}(\kappa=0.8)$. The error bar corresponds to the statistical error (with an integrated luminosity of $800 \mathrm{pb}^{-1}$ ). The rapidity interval is $\Delta \eta=2.6$.

Fig. 2 Bounds on the parameter space $\left(\kappa, M_{W^{\prime}}\right)$ in polarized $p n$ collisions. The shaded areas correspond respectively to the CDF and UA2 excluded regions (in the two-jet channel). The dashed curve is the theoretical upper bound eq. (1), the plain (dotted) curve corresponds to the RHIC upper limit from $A_{L L}^{P V}$ with the same collider parameters as in Fig.1 with $\Delta \eta=2.6(\Delta \eta=1.0)$. 


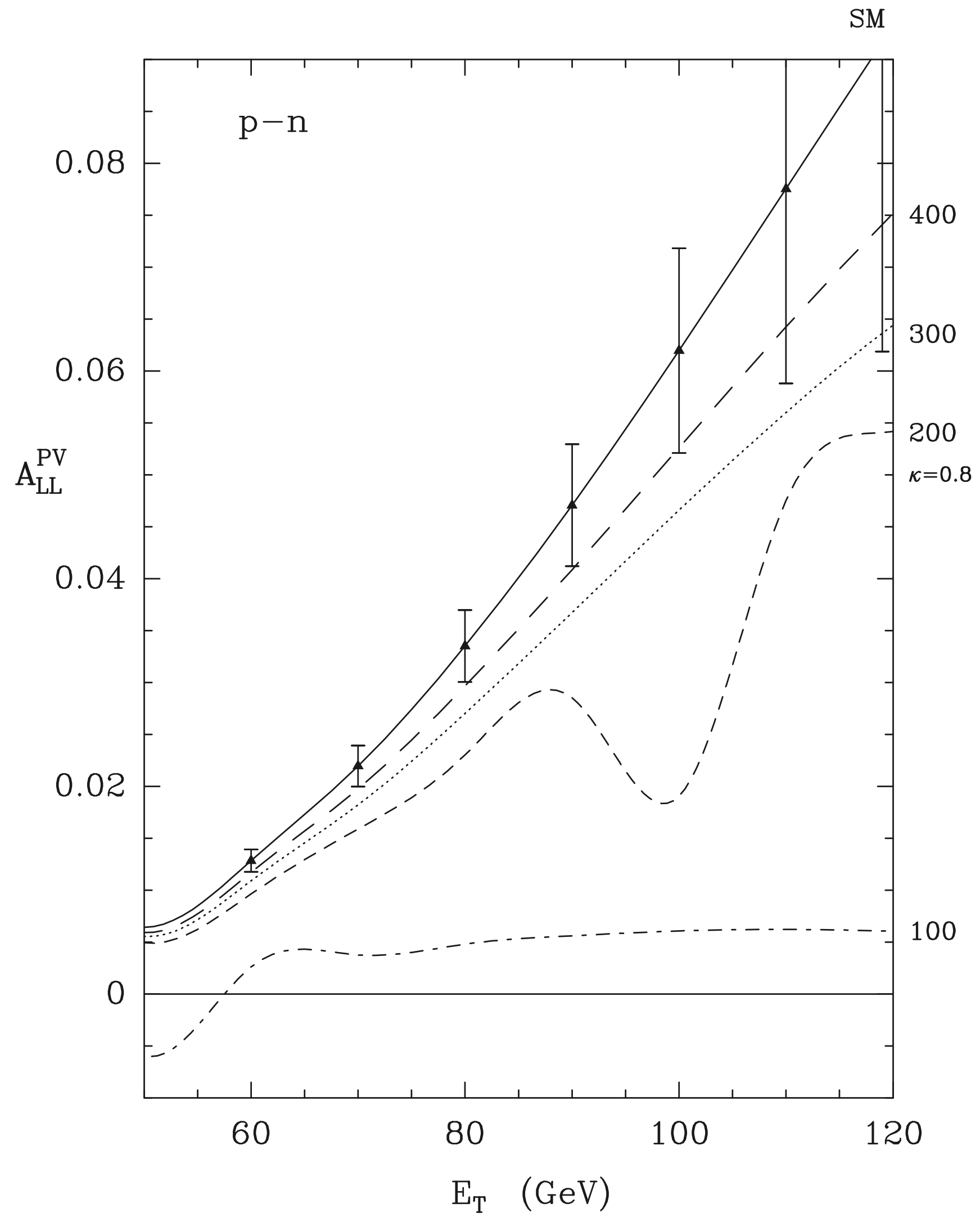

Fig 1 


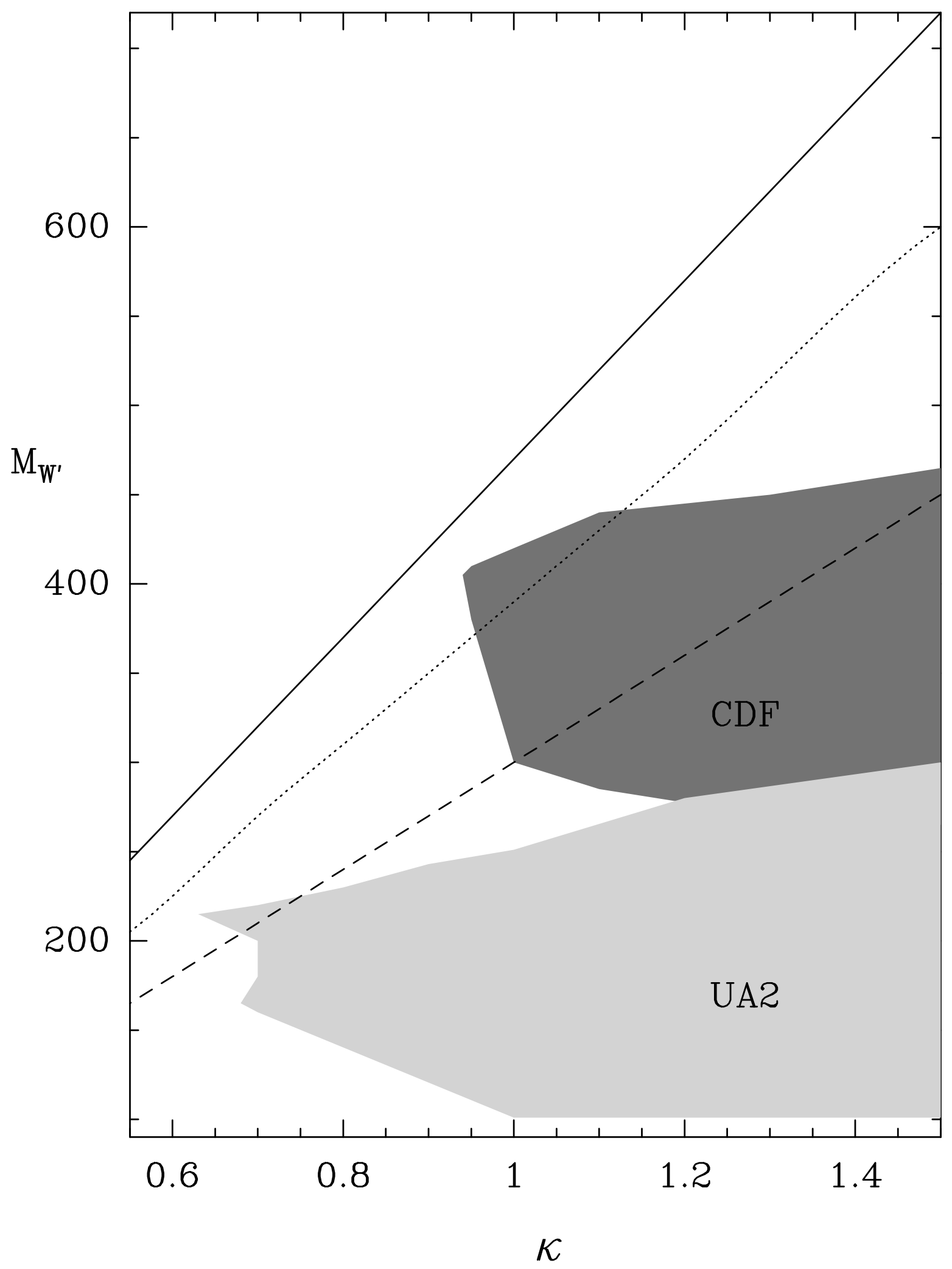

Fig 2 